\title{
Uric Acid as a Potential Peripheral Biomarker for Disease Features in Huntington's Patients
}

\author{
Jody Corey-Bloom ${ }^{1}$, Ameera Haque ${ }^{1}$, Sameer Aboufadel'1, Chase Snell'1, \\ Ryan S. Fischer2, Steven W. Granger' ${ }^{2}$, Douglas A. Granger ${ }^{2,3,4,5,6}$ and \\ Elizabeth A. Thomas ${ }^{3,7 *}$
}

${ }^{1}$ Department of Neurosciences, University of California, San Diego, La Jolla, CA, United States, ${ }^{2}$ Salimetrics, LLC, Carlsbad, CA, United States, ${ }^{3}$ Institute for Interdisciplinary Salivary Bioscience Research, University of California, Irvine, Irvine, CA, United States, ${ }^{4}$ School of Medicine, Johns Hopkins University, Baltimore, MD, United States, ${ }^{5}$ Bloomberg School of Public Health, Johns Hopkins University, Baltimore, MD, United States, ${ }^{6}$ School of Nursing, Johns Hopkins University, Baltimore, MD, United States, ${ }^{7}$ Department of Neuroscience, The Scripps Research Institute, La Jolla, CA, United States

\section{OPEN ACCESS}

Edited by:

Vittorio Maglione, Mediterranean Neurological Institute (IRCCS), Italy

Reviewed by:

Massimo Marano,

Campus Bio-Medico University, Italy

Romina Vuono,

University of Kent, United Kingdom

*Correspondence: Elizabeth A. Thomas bthomas@scripps.edu

Specialty section: This article was submitted to

Neurodegeneration, a section of the journal

Frontiers in Neuroscience

Received: 02 November 2019 Accepted: 20 January 2020

Published: 04 March 2020

Citation:

Corey-Bloom J, Haque A, Aboufadel S, Snell C, Fischer RS, Granger SW, Granger DA and Thomas EA (2020) Uric Acid as a Potential Peripheral Biomarker for Disease Features in Huntington's

Patients. Front. Neurosci. 14:73. doi: 10.3389/fnins.2020.00073
Oxidative stress has long been implicated in the pathophysiology and progression of Huntington's disease (HD). Uric acid (UA) is a naturally occurring antioxidant that is present in the brain and periphery. Growing evidence has implicated UA as a molecular biomarker for several neurodegenerative diseases, most notably Parkinson's disease (PD). In this study, we investigated UA levels in clinical samples from HD patients and normal controls (NCs) and assessed potential relationships between UA levels and disease and clinical data. UA levels were measured in plasma $(n=107)$ and saliva ( $n=178$ ) samples from premanifest (pre-HD) and manifest HD patients and control subjects. Gender effects of UA levels were observed in both biofluids, with male patients showing higher UA levels compared to female patients. Comparisons of UA levels across diagnostic groups, separated by gender, revealed that both plasma and salivary UA levels were significantly lower in female pre-HD and manifest HD patients compared to NCs. Salivary levels of UA were also significantly lower in male manifest HD patients versus controls, but not in plasma. Correlations of peripheral UA levels to clinical data also showed differences according to gender. In male HD patients, both plasma and salivary UA levels were significantly negatively correlated with total functional capacity (TFC), while positive correlations were observed with total motor score (TMS). Female HD patients showed a significant positive correlation between plasma UA levels and TMS, while salivary UA levels from female patients were significantly correlated to disease burden. Finally, in a separate cohort, we show that UA levels are decreased in postmortem prefrontal cortical samples $(n=20)$ from HD subjects compared to matched controls. These findings suggest that decreased levels of UA in the brains of HD patients can be reflected in peripheral fluids, with salivary measures of UA particularly offering significant promise as a potentially relevant, non-invasive biomarker of disease symptoms and burden. Our findings further highlight the impact of sexual dimorphism in $\mathrm{HD}$ pathophysiology.

Keywords: neurodegenerative, biomarker, peripheral, brain, blood, saliva, biofluid 


\section{INTRODUCTION}

Huntington's disease (HD) is an inherited, progressive neurodegenerative disorder caused by a CAG repeat expansion in the $5^{\prime}$ coding region of the Huntington (HTT) gene (Huntington Disease Collaborative Research Group, 1993). Despite enormous progress in our understanding of this disease, the mechanisms connecting mutant huntingtin $(\mathrm{Htt})$ protein with cell death and pathological symptoms remain unclear. Oxidative damage, mitochondrial dysfunction, and impairment in the electron transport chain have been suggested to have important roles in the degenerative process in HD (Browne et al., 1997; Trushina and McMurray, 2007; Franco-Iborra et al., 2018; Zheng et al., 2018). Specifically, past studies have demonstrated enhanced oxidative stress and oxidative DNA damage in peripheral blood from HD patients (Chen et al., 2007), in cultured fibroblasts from HD patients, in HD mouse models (Goula et al., 2009), and in striatal cells derived from HD knock-in mice (Li et al., 2010; Ribeiro et al., 2012). Oxidative damage is not considered to be substantial in the early stages of $\mathrm{HD}$, but rather is proposed to be a major contributor to pathology as the disease progresses (Johri and Beal, 2012). HD symptoms typically appear between the ages of 35 and 55, although juvenile forms of the disease exist, which present before the age of 20 (van Dijk et al., 1986), and the disease progresses over 10-20 years from a presymptomatic state to complete disability and death. The age of onset for HD varies inversely with the length of the disease-causing CAG repeat mutation, with the threshold being between 36 and 39 triplet repeats (Andresen et al., 2007). However, the age of onset and its progression vary considerable among patients, even among patients with identical CAG repeat lengths (Andrew et al., 1993; Wexler et al., 2004; Andresen et al., 2007), warranting the need for biomarkers that might help predict onset. In addition, gender has emerged as another factor that can affect disease outcomes in HD, with women showing worse symptoms and a faster rate of progression (Dorner et al., 2007; Zielonka et al., 2013). In fact, there are several gender-related differences that have been reported in other neurodegenerative diseases as well, which have enhanced our current understanding of the impact of sexual dimorphism in neurological diseases and the implications for preventive and therapeutic outcomes (Zagni et al., 2016; Ullah et al., 2019).

Uric acid (UA), an endogenous compound that is formed as the end-product of purine metabolism, is a powerful antioxidant in the CNS and periphery. In fact, UA is the most abundant natural antioxidant in human blood. Although imbalanced UA levels have a long-standing association with medical conditions such as gout, UA also affects the central nervous system and has been specifically been linked to neurological conditions (Bowman et al., 2010; Fang et al., 2013). With respect to neurodegenerative diseases, the interest in UA was initially sparked by evidence of oxidative damage in another movement disorder, Parkinson's disease (PD). Previous work in PD has demonstrated low levels of UA in postmortem substantia nigra (Church and Ward, 1994; McFarland et al., 2013), as well as in blood from PD patients (Davis et al., 1996; Schlesinger and Schlesinger, 2008). Since these early observations, accumulating epidemiological studies, laboratory data, preclinical models, and early clinical trial results have provided substantial support for a neuroprotective role for $\mathrm{UA}$ and its potential as a disease biomarker for PD (Constantinescu and Zetterberg, 2011; Chen et al., 2012; Crotty et al., 2017). In particular, UA has been suggested as a promising biomarker of reduced risk and milder progression in PD (Schlesinger and Schlesinger, 2008; Paganoni and Schwarzschild, 2017; Wen et al., 2017).

Because oxidative stress represents a shared pathological mechanism, not only between PD and HD but also across several neurodegenerative diseases, we hypothesized that UA could also serve as a potentially relevant biomarker in HD. Hence, in this study, we measured UA levels in peripheral fluids and postmortem brain samples from HD patients and assessed their potential relationships with disease and clinical data. We include saliva in this study as a non-invasive alternative to blood, and a biofluid that has the potential to expand biomarker measurements in diverse settings.

\section{MATERIALS AND METHODS}

\section{Human Subjects}

This study was approved by the University of California, San Diego (UCSD) Institutional Review Board in accordance with the requirements of the Code of Federal Regulations on the Protection of Human Subjects. Informed consent from all subjects was obtained before their participation in accordance with the Declaration of Helsinki. Patients were recruited from the UCSD HDSA Center of Excellence. HD patient criteria included a definitive diagnosis of HD with family history and an expanded trinucleotide CAG repeat of 40 or more. Normal controls (NCs) had no reported history of neurological conditions, psychiatric disorders, or gout, and no use of psychoactive substances or medications. Demographic information was collected at the time of saliva collection, including sex, age, CAG repeat length, and age of onset. This information is provided in Table 1. Patients were assessed for cognitive and motor function using the Mini-Mental State Examination (MMSE; score range, 0-30) (Gluhm et al., 2013), the Unified Huntington's Disease Rating Scale (UHDRS) total motor score (TMS; range, $0-124$ ), and total functional capacity (TFC; range, $0-13$ ). Overall genetic or disease burden was assessed using the disease burden score (DBS). These data are summarized in Supplementary Table S1.

\section{Plasma Collection}

Blood from consenting HD patients and controls was drawn by venipuncture into $2 \mathrm{ml}$ lavender/ethylenediaminetetraacetic acid (EDTA) tubes. EDTA/whole blood was mixed well by inversion and spun at $900 \times g$ for $15 \mathrm{~min}$. The top plasma layer was transferred into $4 \times 1 \mathrm{ml}$ aliquots and snap frozen and stored at $-80^{\circ} \mathrm{C}$. All subjects who provided a plasma sample also provided a saliva sample.

\section{Saliva Collection}

All donors were asked to refrain from smoking, eating, drinking, or oral hygiene procedures for at least $1 \mathrm{~h}$ before sample 
TABLE 1 | Summary of subjects used for these studies.

\begin{tabular}{|c|c|c|c|c|}
\hline & HD & Pre-HD & NC & Total no. \\
\hline \multicolumn{5}{|l|}{ Plasma } \\
\hline Number of patients & 38 & 31 & 38 & 107 \\
\hline Female/male & $22: 16$ & $18: 13$ & $18: 20$ & $58: 49$ \\
\hline Average age (years) & $59.5 \pm 13.1$ & $43.7 \pm 13.8$ & $59.9 \pm 14.3$ & \\
\hline Average CAG repeat & $42.7 \pm 2.8$ & $41.3 \pm 1.9$ & NA & \\
\hline \multicolumn{5}{|l|}{ Saliva } \\
\hline Number of patients & 45 & 49 & 84 & 178 \\
\hline Female/male & $29: 16$ & $26: 23$ & $41: 43$ & $96: 82$ \\
\hline Average age (years) & $56.5 \pm 13.4$ & $44.8 \pm 12.2$ & $52.9 \pm 16.02$ & \\
\hline Average CAG repeat & $43.5 \pm 2.8$ & $41.0 \pm 1.7$ & NA & \\
\hline \multicolumn{5}{|l|}{ Postmortem brain } \\
\hline Number of subjects & 10 & 10 & & 20 \\
\hline Female/male & $3: 7$ & $3: 7$ & & $6: 14$ \\
\hline Average age (years) & $59.6 \pm 4.33$ & $60.0 \pm 4.32$ & & \\
\hline Average PMl & $13.5 \pm 5.08$ & $16.8 \pm 9.1$ & & \\
\hline
\end{tabular}

PMI, postmortem interval; HD, Huntington's disease; Pre-HD, premanifest; NC, normal controls.

collection and then rinsed their mouth thoroughly with water 15$20 \mathrm{~min}$ before sample collection. Saliva samples were collected between 10:00 AM and 4 PM using the passive drool method according to previously established protocols (Granger et al., 2012). Roughly 2-3 ml of unstimulated whole saliva was obtained. Besides the patients who provided a plasma sample, an additional group of patients provided a saliva-only sample. Samples were immediately frozen at $-20^{\circ} \mathrm{C}$ at the time of collection and then stored at $-80^{\circ} \mathrm{C}$. At the time of use, saliva samples were thawed and centrifuged at $10,000 \times g$ for $10 \mathrm{~min}$ at $4^{\circ} \mathrm{C}$ to remove insoluble material and cellular debris. Supernatants were collected and used for all assays.

\section{Postmortem Brain Samples}

Prefrontal cortical tissue was obtained postmortem from subjects with $\mathrm{HD}$ cases and age-matched NCs with no history of psychiatric illness. Tissues were obtained from the National Institutes of Health (NIH) NeuroBioBank ${ }^{1}$. All HD cases showed a moderately to severely atrophied cortical tissue with pathological grades 2-4. Exact CAG repeat numbers were not available. Details such as Vonsattel grading, age, gender, and postmortem delay can be found in Table 1. Frozen tissue samples $(\sim 1 \mathrm{~g})$ were homogenized in lysis buffer and then centrifuged at $10,000 \times g$ at $4^{\circ} \mathrm{C}$ for $20 \mathrm{~min}$. Supernatant fractions were used for UA assays below. Total protein levels in each sample were quantified by the bicinchoninic acid (BCA) protein assay (Abcam). Brain samples were obtained from a separate cohort of subjects than those providing plasma and/or saliva samples above.

\section{UA Measurements}

Uric acid levels in all samples were measured using a commercially available colorimetric enzymatic reaction kit

${ }^{1}$ https://neurobiobank.nih.gov/
(Catalog No. 1-3802, Salimetrics, CA, United States). The amount of UA in the sample was assessed by measuring the production of red chromogen during an enzymatic reaction process. All assays were performed in duplicate by operators blinded to the clinical state of the participant. The assay used $10 \mu \mathrm{l}$ of sample per well and had a range of sensitivity from 0.78 to $25 \mathrm{mg} / \mathrm{dl}$.

\section{Statistics}

The distribution of the data values in brain samples and each peripheral fluid was tested for normality using the KolmogorovSmirnov normality test to determine whether parametric or non-parametric tests would be used for correlation variables. Outlier values were determined using either the Grubb's test for sex and age comparisons or the Iglewicz and Hoaglin's robust test for multiple outliers (two-sided test; $z=3.5$ ) for diagnostic comparisons, resulting in the removal of two datapoints for the plasma samples and four datapoints for the saliva samples. A oneway analysis of covariance (ANCOVA) was used to determine if differences in diagnostic groups were associated with age or gender. Group differences separated by sex were determined by one-way ANOVA, followed by Bonferroni or Tukey's multiple comparison test. Linear regression analysis (Pearson or Spearman correlation) was used to compare UA levels against disease and clinical variables. Differences between sex and postmortem interval (brain samples only) were determined using Student's $t$-test (unpaired; two-tailed). Statistical analyses were performed using JASP STATs and Prism.

\section{RESULTS}

\section{Plasma UA in HD Patients}

We measured UA concentrations in plasma from patients with manifest HD $(n=38)$, premanifest HD (designated "preHD") ( $n=31)$, and healthy NC individuals with no history of neurological or psychiatric disorders $(n=38)$ (Table 1). There was no significant difference in plasma UA levels between genders in control subjects, although levels were significantly higher in male compared to female patients in pre-HD and HD subjects (Figure 1A) consistent with previous studies (Andreadou et al., 2009; Kivity et al., 2013; Riis et al., 2018; Yang et al., 2019). Gender ratios across the three groups of subjects were not significantly different. The pre-HD group was statistically significantly younger than both the controls and manifest HD groups (ANOVA $p<0.001$ ); however, when both age and gender were considered as covariates in determining the difference between diagnostic groups using ANCOVA, the age effect was not significant $(p<0.193)$, with the gender effect remaining $(p<0.006)$. Accordingly, linear correlation revealed no significant effects of age on UA levels (Figure 1B).

Owing to the significant gender effect, we compared UA levels across diagnostic groups in male and female HD patients separately. Using ANOVA, we found that female subjects showed significantly lower levels of UA in both pre-HD and manifest HD patients versus NCs (ANOVA $p<0.014 ; p<0.05$ for NC vs. pre-HD and NC vs. HD; Figure 2A). Similar decreases were not observed in male patients (ANOVA $p<0.469$; Figure 2B). 

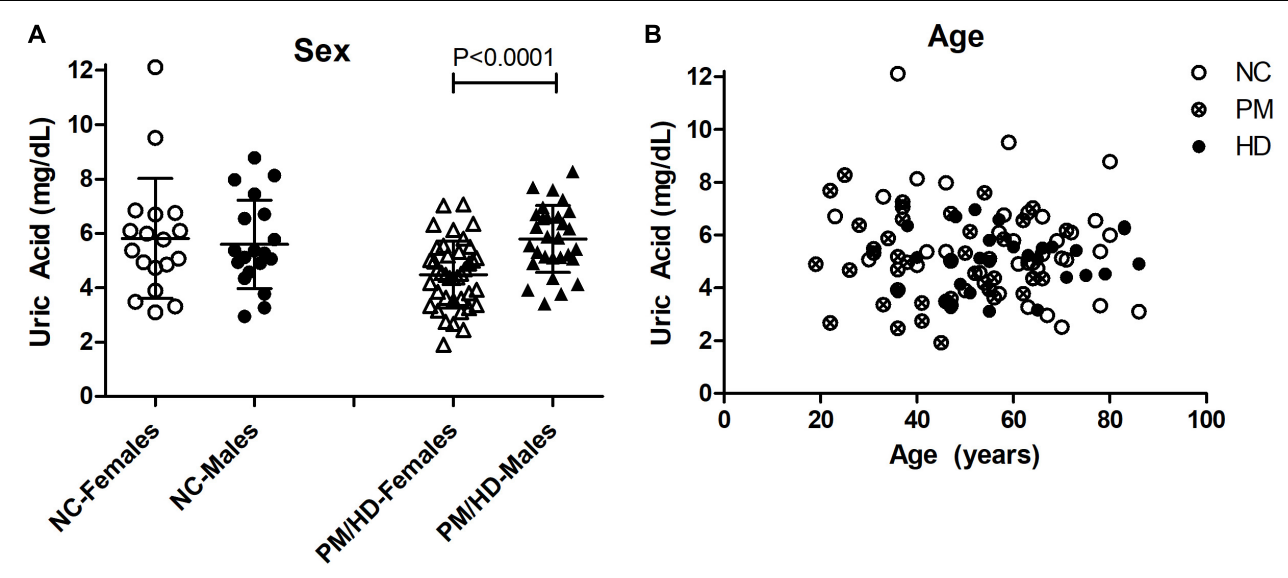

FIGURE 1 | Gender and age effects of uric acid (UA) in plasma from Huntington's disease (HD) patients and normal controls (NC). (A) Significant differences in gender were determined by Student's $t$-test, unpaired; two-tailed; $p<0.0001$. The HD patients include pre-HD (PM) and manifest HD patients. (B) Plasma UA levels were not significantly associated with age in any group.

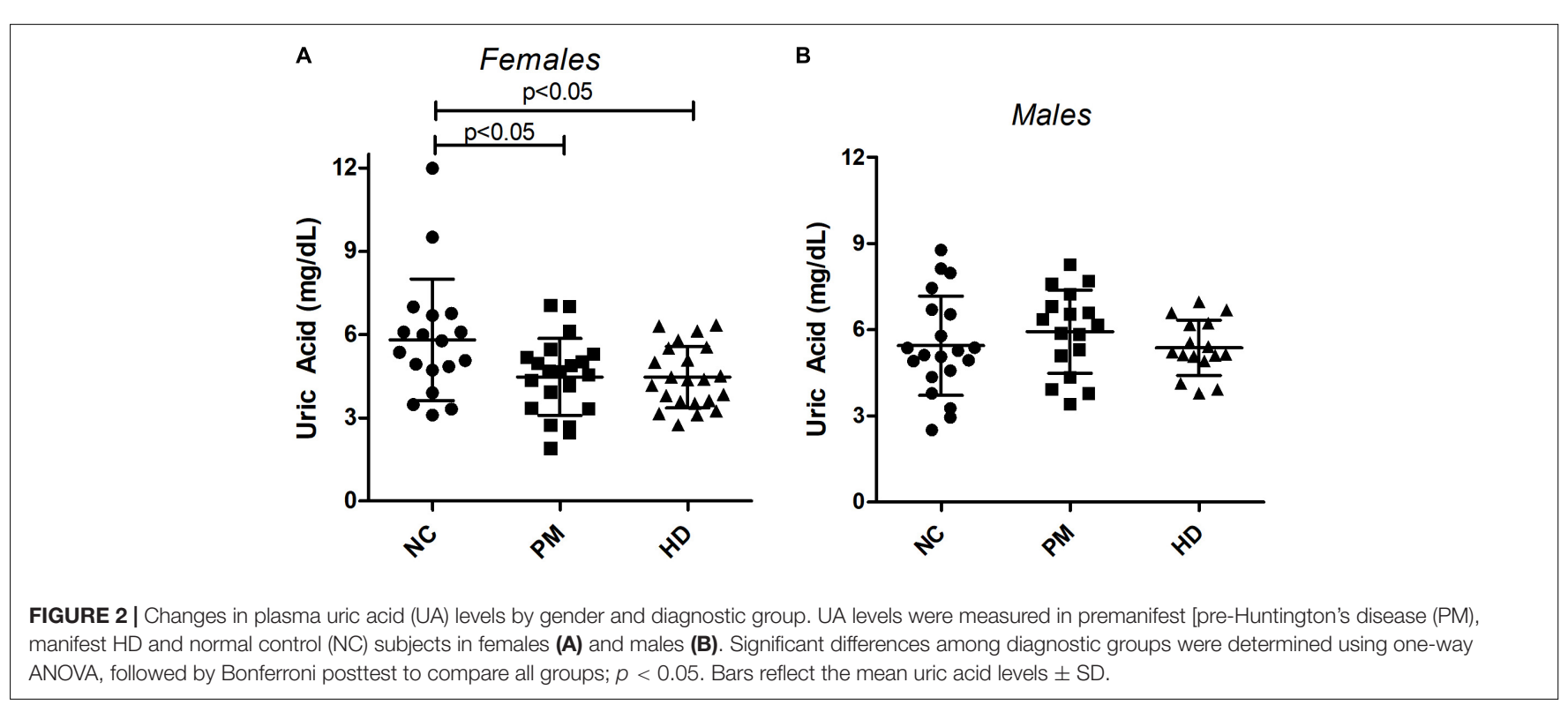

We next correlated UA levels with clinical data, keeping the gender distinction. In male manifest HD patients, plasma UA levels were significantly negatively correlated with TFC (Pearson $r=-0.600 ; p=0.014)$ and positively correlated with TMS from the UHDRS (Pearson $r=0.644 ; p=0.007$ ) (Table 2). In female manifest HD patients, plasma UA levels were positively correlated with TMS (Pearson $r=0.656 ; p=0.005$ ), similar to the effect observed in male patients (Table 2). No significant correlations were detected between plasma UA levels and clinical or disease metrics in pre-HD patients of either gender.

\section{Measures of UA in Saliva From HD Patients}

We next assessed UA levels in saliva from pre-HD patients $(N=49)$, HD patients $(n=45)$, and control subjects $(n=84)$ (Table 1). We found that salivary UA levels were significantly higher in male compared to female patients in both cohorts (Figure 3A), consistent with previous studies on saliva (Riis et al., 2018). Similar to the plasma cohort, the pre-HD patients were statistically significantly younger than both the controls and manifest HD groups (ANOVA $p<0.0001$ ); however, age was not considered a significant covariate in determining the difference between diagnostic groups using ANCOVA $(p<0.350)$. Accordingly, linear correlation revealed no significant effects of age on salivary UA levels in any diagnostic group (Figure 3B).

Owing to the significant gender effect, we compared salivary UA levels across diagnostic groups in male and female HD patients separately. We found that salivary UA levels were significantly lower in female pre-HD and HD patients compared to NCs, with nearly a $40 \%$ reduction in UA concentration detected in HD patients (ANOVA $p<0.0048$; $p<0.05$ for NC vs. pre-HD and $\mathrm{NC}$ vs. HD, Figure $\mathbf{4 A}$ ). In male patients, UA levels were significantly lower in manifest HD patients compared 
TABLE 2 | Correlations between plasma uric acid and clinical and disease data in female and male HD patients.

\begin{tabular}{|c|c|c|c|c|c|c|c|c|}
\hline & \multicolumn{4}{|c|}{ Female } & \multicolumn{4}{|c|}{ Male } \\
\hline & \multicolumn{2}{|c|}{ Premanifest HD } & \multicolumn{2}{|c|}{ Manifest HD } & \multicolumn{2}{|c|}{ Premanifest HD } & \multicolumn{2}{|l|}{ Manifest HD } \\
\hline & $\begin{array}{l}\text { Pearson/ } \\
\text { Spearman } \\
\text { rho value }\end{array}$ & $p$-Value & $\begin{array}{l}\text { Pearson/ } \\
\text { Spearman } \\
\text { rho value }\end{array}$ & $p$-Value & $\begin{array}{l}\text { Pearson/ } \\
\text { Spearman } \\
\text { rho value }\end{array}$ & $p$-Value & $\begin{array}{l}\text { Pearson/ } \\
\text { Spearman } \\
\text { rho value }\end{array}$ & $p$-Value \\
\hline DBS & 0.439 & 0.077 & -0.012 & 0.944 & 0.306 & 0.310 & 0.171 & 0.526 \\
\hline TFC & 0.274 & 0.071 & -0.133 & 0.426 & -0.207 & 0.498 & -0.600 & 0.014 \\
\hline MMSE & 0.231 & 0.959 & -0.093 & 0.579 & -0.152 & 0.620 & -0.179 & 0.508 \\
\hline TMS & 0.075 & 0.690 & 0.656 & 0.005 & 0.029 & 0.926 & 0.644 & 0.007 \\
\hline CAG repeat no. & 0.533 & 0.057 & -0.087 & 0.621 & 0.380 & 0.353 & -0.064 & 0.820 \\
\hline Age of Onset & NA & NA & 0.296 & 0.180 & NA & NA & 0.171 & 0.527 \\
\hline Disease duration & NA & NA & 0.140 & 0.402 & NA & NA & 0.100 & 0.714 \\
\hline
\end{tabular}

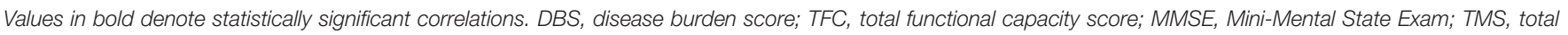
motor symptom score.

to controls and pre-HD subjects (ANOVA $p<0.0001 ; p<0.001$ for NC vs. pre-HD and NC vs. HD, respectively, Figure 4B).

Comparing to clinical data, we found that salivary UA levels in male HD patients showed the same effects as plasma UA levels, being significantly negatively correlated with TFC (Pearson $r=-0.617 ; p=0.012$ ) and positively correlated with TMS (Pearson $r=0.627 ; p=0.009$ ) (Table 3). In female HD patients, salivary UA levels were significantly correlated with DBS (Pearson $r=-0.434 ; p=0.009$ ), CAG repeat length (Spearman $r=-0.397 ; p=0.018$ ). No significant correlations were observed between salivary UA levels and any clinical or disease metric in pre-HD patients of either gender, consistent with the findings from blood.

\section{Plasma UA Levels Versus Salivary UA Levels}

All subjects who donated a plasma sample also provided a saliva sample; hence, we could directly compare levels of UA in both peripheral fluids in the same individuals. In both male and female normal subjects, plasma levels of UA were higher than those found in saliva (Student's $t$-test; $p<0.0001$ ), consistent with previous studies (Riis et al., 2018). We next examined whether levels of UA in blood correlated with those detected in saliva in all pre-HD and HD patients and found a significant correlation between UA levels in plasma versus saliva (Spearman $r=0.495 ; p<0.0001$ ) (Figure 5), suggesting that salivary UA levels originate, at least in part, from blood sources.

\section{Uric Acid Levels in HD Postmortem Brain}

In a separate study, we measured UA levels in prefrontal cortex obtained postmortem from $10 \mathrm{HD}$ patients and 10 healthy age-matched NCs. The demographic data for these subjects are shown in Table 1. There were no significant differences in mean age, postmortem interval, or sex ratios between the $\mathrm{HD}$ and control groups. UA measurements were normalized by total protein levels in each sample to account for differences in starting amounts of tissue. Regression analyses revealed no significant correlations between UA and age or postmortem interval in either group (Supplementary Table S2), although a trend toward a significant correlation with age was observed in the control group (Supplementary Table S2). Comparing between diagnostic groups revealed a significant decrease in UA levels in prefrontal cortex from $\mathrm{HD}$ patients compared to NCs $(16.8 \pm 5.6$ vs. $34.3 \pm 21.4 \mathrm{ng} / \mathrm{mg}$ total protein) (Figure 6). This difference was not affected by sex or age as determined by ANCOVA ( $p=0.270$ and $p=0.337$ for sex and age, respectively).

\section{DISCUSSION}

Despite accumulating evidence for enhanced oxidative stress and oxidative damage in HD (Chen et al., 2007; Goula et al., 2009; Li et al., 2010; Ribeiro et al., 2012), the major pathways and the sequence of oxidative stress-associated events in HD pathophysiology are poorly understood. Nonetheless, as an endogenous antioxidant, UA represents a potentially relevant biomarker for studies in HD. In this study, we show that UA levels are significantly lower in the plasma and saliva of HD patients compared to healthy controls. This decrease in UA was also detected in postmortem brain samples from HD patients, which is consistent with a previous study that found lower UA levels in postmortem frontal lobe and striatum from HD subjects using high-resolution mass spectrometry (Graham et al., 2016). However, our findings further demonstrate that decreased UA concentrations are also detected in peripheral fluids, including the non-invasive biofluid, saliva. This could possibly reflect a reduced antioxidant capacity in HD that is manifested throughout the body.

Importantly, our results highlight important gender differences in UA levels and the utility for UA to serve as a potential disease biomarker. There are a number of genderrelated differences that have been reported in neurodegenerative diseases (Ullah et al., 2019), which have made it necessary to incorporate sex as a biological factor in experimental and clinical studies. Importantly, such studies have enhanced the current understanding of the impact of sexual dimorphism in neurological diseases (Zagni et al., 2016; Ullah et al., 2019). In our studies, we first found that UA levels were higher in male 

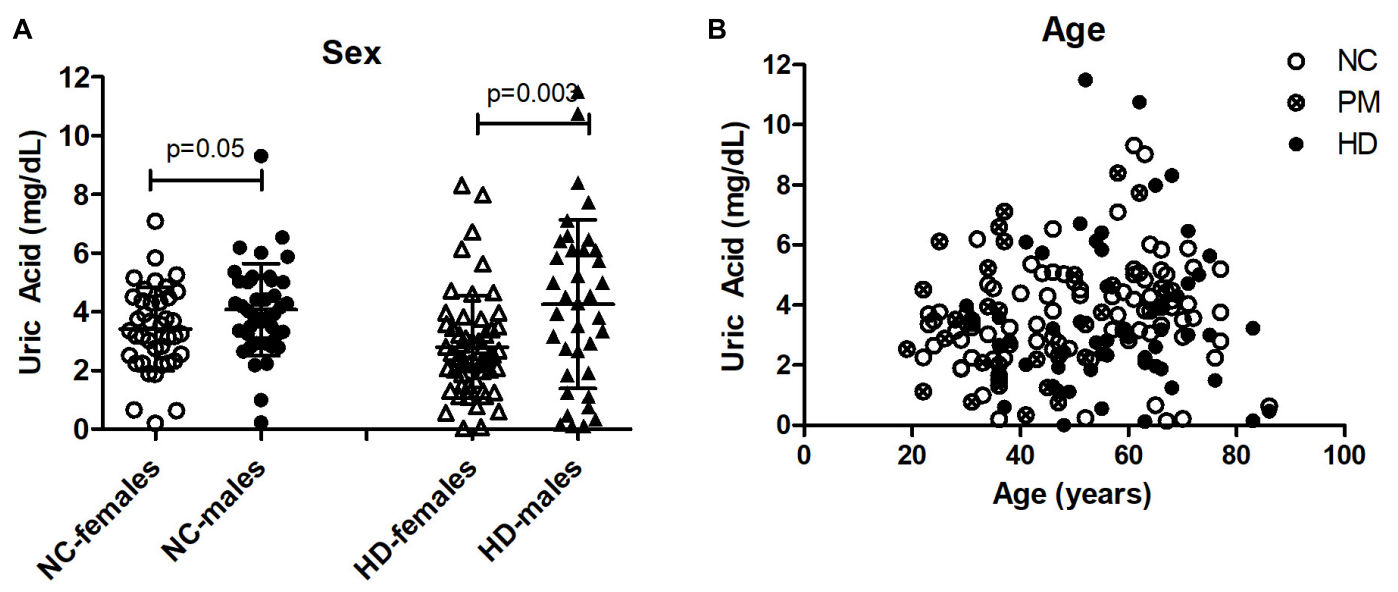

FIGURE 3 | Gender and age effects of uric acid (UA) in saliva from Huntington's disease (HD) patients and normal controls (NC). (A) UA levels were significantly higher in males compared to females in both $\mathrm{NC}$ and HD groups. The HD group also included pre-HD patients (PM). Significant differences were determined by Student's $t$-test, unpaired; two-tailed; $p=0.05 ; p=0.003$. (B) No significant effects of age on salivary UA levels were observed in any group.
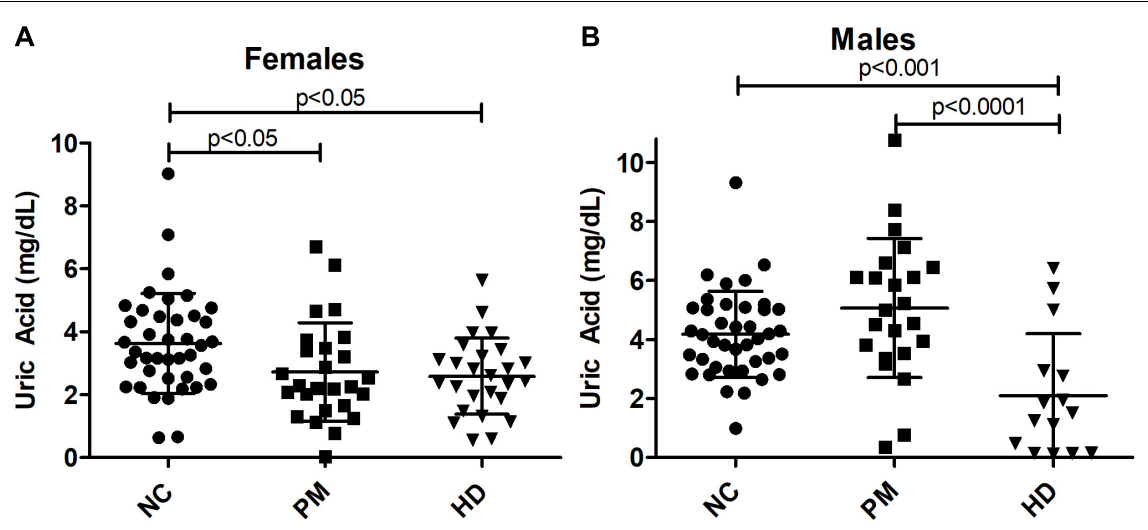

FIGURE 4 | Alterations in salivary uric acid (UA) levels according to diagnostic group in females (A) and males (B). Uric acid levels are shown according to diagnostic group, premanifest [pre-Huntington's disease (PM), manifest HD (HD), and normal controls (NC). Significant differences among diagnostic groups were determined using one-way ANOVA, followed by Bonferroni posttest to compare all groups: $p<0.05, p<0.001, p<0.0001$, as shown. Bars reflect the mean uric acid levels $\pm \mathrm{SD}$.

TABLE 3 | Correlations between salivary uric acid and clinical and disease data in female and male HD patients.

\begin{tabular}{|c|c|c|c|c|c|c|c|c|}
\hline & \multicolumn{4}{|c|}{ Females } & \multicolumn{4}{|c|}{ Males } \\
\hline & \multicolumn{2}{|c|}{ Premanifest HD } & \multicolumn{2}{|c|}{ Manifest HD } & \multicolumn{2}{|c|}{ Premanifest HD } & \multicolumn{2}{|c|}{ Manifest HD } \\
\hline DBS & 0.257 & 0.186 & -0.435 & 0.009 & -0.020 & 0.943 & 0.444 & 0.129 \\
\hline MMSE & -0.234 & 0.221 & 0.209 & 0.236 & -0.412 & 0.113 & -0.339 & 0.257 \\
\hline TMS & 0.157 & 0.418 & -0.090 & 0.605 & 0.019 & 0.945 & 0.627 & 0.009 \\
\hline CAG repeat \# & 0.052 & 0.848 & -0.397 & 0.018 & -0.004 & 0.990 & 0.538 & 0.058 \\
\hline Age of Onset & NA & NA & 0.186 & 0.285 & NA & NA & -0.379 & 0.202 \\
\hline Disease duration & NA & NA & 0.104 & 0.553 & NA & NA & -0.111 & 0.718 \\
\hline
\end{tabular}

DBS, disease burden score; TFC, total functional capacity score. MMSE, mini-mental state exam; TMS, total motor symptom score. Values shown in bold denote statistically significant correlations. 


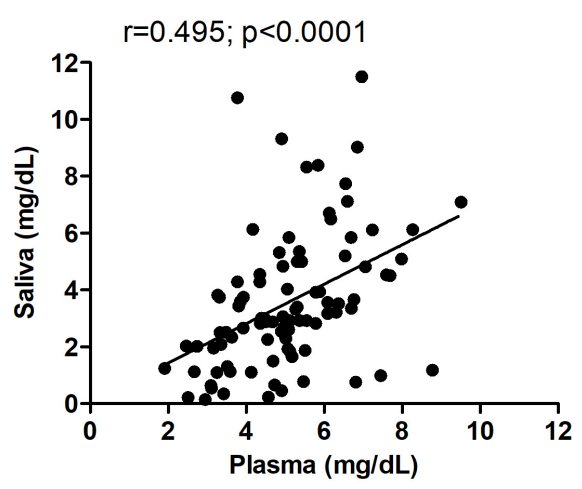

FIGURE 5 | Correlation between plasma and salivary levels of uric acid (UA). The correlation between plasma and salivary levels of UA was determined using Spearman correlation analysis. Pearson $r=0.495 ; p<0.0001$. The same subjects provided paired saliva and plasma samples; however, for some subjects, the saliva and plasma were collected on different days spaced up to a week apart.

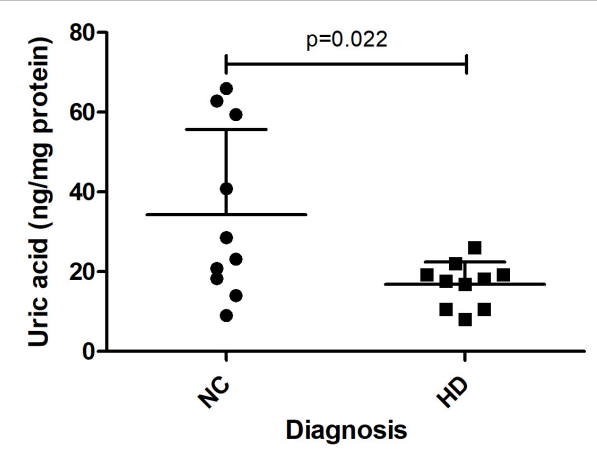

FIGURE 6 | Uric acid (UA) levels in postmortem prefrontal cortex from Huntington's disease (HD) subjects and matched controls. UA levels, corrected for total protein, were measured in prefrontal cortical samples from $n=10 \mathrm{HD}$ patients and $n=10$ matched normal controls (NC). Data points reflect mean $+\mathrm{SD}$. Difference in UA concentrations in HD versus normal controls was determined using Student's $t$-test (unpaired, two-tailed), $p=0.022$.

compared to female patients in all of the biospecimens we tested. This is consistent with several past studies, where it has been shown that men have higher levels of UA than women in blood and saliva (Andreadou et al., 2009; Kivity et al., 2013; Riis et al., 2018; Yang et al., 2019). The reason for this difference is not clear, but possible explanations include alcohol consumption, which is higher in men, and the influence of estrogens to lower UA (Jung et al., 2018; Yang et al., 2019). Next, we found gender differences in the ability of UA to distinguish diagnostic groups. In female patients, plasma and salivary levels of UA were significantly lower in both pre-HD and manifest HD patients, while in male patients, UA concentrations were only lower in the saliva of manifest HD patients. The higher levels found in the saliva from male patients, in general, might mask a potential disease effect of UA tested here.
Or there may be a true difference in the role of UA in HD pathology in male vs. female patients. For example, a study examining the relationship between gender and progression of the disease showed that gender played a significant role in the neuropathological changes and phenotypical expression in HD (Dorner et al., 2007). Another study also found a gender influence in disease severity with women displaying poorer motor and functional UHDRS scores (Zielonka et al., 2013). Hence, it is possible that disease-propagating mechanisms are different in men and women, and our studies would suggest an involvement of oxidative stress in these mechanisms. We found that in male HD patients, both plasma and salivary UA levels were significantly positively correlated with TMS and negatively correlated with TFC. The direction of these correlations might appear at odds with the decreased levels of UA detected in HD patients compared to NCs, which would suggest that a reduced antioxidant capacity is detrimental. However, within the HD population, our results would suggest that higher UA levels are more damaging. It is known that too much UA can have negative consequences, such as proinflammatory effects, associated with increased cytokines (Fang et al., 2013). This is consistent with the literature demonstrating increase proinflammatory markers, including interleukin 6 and $1 \mathrm{~B}$, in the plasma of premanifest $\mathrm{HD}$ patients and in presymptomatic HD mice (Dalrymple et al., 2007; Chang et al., 2015; Politis et al., 2015).

Our findings in plasma are consistent with past literature in other neurodegenerative disorders, where lower blood and brain levels of UA have been reported in PD, ALS, and Alzheimer's disease patients (Church and Ward, 1994; Constantinescu and Zetterberg, 2011; Chen et al., 2012; Crotty et al., 2017). In PD, correlations were observed between higher serum levels of UA and not only a lower risk of developing the disease but also a slower rate of disease progression (Schlesinger and Schlesinger, 2008; Paganoni and Schwarzschild, 2017; Wen et al., 2017). Interestingly, studies in PD have also reported differences in men and women, with one study showing correlations between serum UA levels and disease duration in patients with PD only in men (Andreadou et al., 2009). Other studies have implicated serum UA as a biomarker for coronary artery disease only in women (Sun et al., 2019).

Our findings in saliva are novel. Biomarker research in saliva has grown over the past few years (Corey-Bloom et al., 2018), largely due to its non-invasive nature and ease of collection in any setting. Here, we have shown that the UA data in saliva mimic several features of plasma and that levels of salivary UA are correlated with those found in plasma. This would suggest that at least some of the UA present in saliva originates from blood sources. However, salivary levels of UA could also arise from leukocytes and buccal cells present in saliva, or the salivary glands themselves, which are innervated by the glossopharyngeal and facial nerves, suggesting potential neural release of UA in saliva.

Potential confounds of our study could be the effects of diet and stress, which could differ between patients and controls. It is known that UA levels can vary according to diet (Zgaga et al., 2012; Towiwat and Li, 2015), and stress and strenuous exercise have been shown to elevate UA (Gonzalez et al., 2008). 
However, previous studies have demonstrated that, while there is some "state specific" variation in salivary UA levels, the majority of the variance in salivary UA can be attributable to a "trait-like" stable component of the variance (Riis et al., 2018). In addition, the potential effects of medications should be considered in future studies on saliva. For example, some medications can affect UA levels (Caspi et al., 2000) or can cause xerostomia, reducing salivary output.

The utility of salivary measures to reveal information about HD disease features is especially exciting, given the non-invasive nature of saliva collection, although this is a new field with respect to neurological disorders. UA could be an important potential biomarker of disease symptoms and genetic burden, albeit in a gender-specific manner. The fact that the decrease in UA levels in the CNS can be revealed in peripheral fluids is especially exciting and should encourage future studies investigating UA levels in cerebrospinal fluid (CSF) from HD patients.

\section{DATA AVAILABILITY STATEMENT}

All clinical data generated for this study are included in the article/Supplementary Material.

\section{ETHICS STATEMENT}

The studies involving human participants were reviewed and approved by the University of California, San Diego Institutional Review Board in accordance with the requirements of the Code

\section{REFERENCES}

Andreadou, E., Nikolaou, C., Gournaras, F., Rentzos, M., Boufidou, F., Tsoutsou, A., et al. (2009). Serum uric acid levels in patients with Parkinson,s disease: their relationship to treatment and disease duration. Clin. Neurol. Neurosurg 111, 724-728. doi: 10.1016/j.clineuro.2009.06.012

Andresen, J. M., Gayan, J., Djousse, L., Roberts, S., Brocklebank, D., Cherny, S. S., et al. (2007). The relationship between CAG repeat length and age of onset differs for Huntington's disease patients with juvenile onset or adult onset. Ann. Hum. Genet. 71(Pt 3), 295-301. doi: 10.1111/j.1469-1809.2006. 00335.x

Andrew, S. E., Goldberg, Y. P., Kremer, B., Telenius, H., Theilmann, J., Adam, S., et al. (1993). The relationship between trinucleotide (CAG) repeat length and clinical features of Huntington's disease. Nat. Genet. 4, 398-403. doi: 10.1038/ng0893-398

Bowman, G. L., Shannon, J., Frei, B., Kaye, J. A., and Quinn, J. F. (2010). Uric acid as a CNS antioxidant. J. Alzheimers. Dis. 19, 1331-1336. doi: 10.3233/JAD-20101330

Browne, S. E., Bowling, A. C., MacGarvey, U., Baik, M. J., Berger, S. C., Muqit, M. M., et al. (1997). Oxidative damage and metabolic dysfunction in Huntington's disease: selective vulnerability of the basal ganglia. Ann. Neurol. 41, 646-653. doi: 10.1002/ana.410410514

Caspi, D., Lubart, E., Graff, E., Habot, B., Yaron, M., and Segal, R. (2000). The effect of mini-dose aspirin on renal function and uric acid handling in elderly patients. Arthritis Rheum. 43, 103-108. doi: 10.1002/1529-0131(200001)43: $1<103$ ::aid-anr13>3.0.co;2-c

Chang, K. H., Wu, Y. R., Chen, Y. C., and Chen, C. M. (2015). Plasma inflammatory biomarkers for Huntington's disease patients and mouse model. Brain Behav. Immun. 44, 121-127. doi: 10.1016/j.bbi.2014.09.011

Chen, C. M., Wu, Y. R., Cheng, M. L., Liu, J. L., Lee, Y. M., Lee, P. W., et al. (2007). Increased oxidative damage and mitochondrial abnormalities in the peripheral of Federal Regulations on the Protection of Human Subjects. The patients/participants provided their written informed consent to participate in this study.

\section{AUTHOR CONTRIBUTIONS}

JC-B designed the research plan, recruited all subjects for this study, and collected demographic and clinical data from HD patients. AH collected and organized clinical data, performed statistical analyses, and collected saliva samples from HD patients. SA collected clinical data from HD patients. CS collected saliva samples from HD patients. RF performed the experiments. SG provided reagents. DG provided reagents. ET designed the research plan, performed experiments, performed statistical analyses, and wrote the manuscript.

\section{FUNDING}

This study was funded by grants from the National Institutes of Health (NS111655 to ET).

\section{SUPPLEMENTARY MATERIAL}

The Supplementary Material for this article can be found online at: https://www.frontiersin.org/articles/10.3389/fnins. 2020.00073/full\#supplementary-material

blood of Huntington's disease patients. Biochem. Biophys. Res. Commun. 359, 335-340. doi: 10.1016/j.bbrc.2007.05.093

Chen, X., Wu, G., and Schwarzschild, M. A. (2012). Urate in Parkinson's disease: more than a biomarker? Curr. Neurol. Neurosci. Rep. 12, 367-375. doi: 10.1007/ s11910-012-0282-7

Church, W. H., and Ward, V. L. (1994). Uric acid is reduced in the substantia nigra in Parkinson's disease: effect on dopamine oxidation. Brain Res. Bull. 33, 419-425. doi: 10.1016/0361-9230(94)90285-2

Constantinescu, R., and Zetterberg, H. (2011). Urate as a marker of development and progression in Parkinson's disease. Drugs Today 47, 369-380. doi: 10.1358/ dot.2011.47.5.1591834

Corey-Bloom, J., Haque, A. S., Park, S., Nathan, A. S., Baker, R. W., and Thomas, E. A. (2018). Salivary levels of total huntingtin are elevated in Huntington's disease patients. Sci. Rep. 8:7371. doi: 10.1038/s41598-018-25095-3

Crotty, G. F., Ascherio, A., and Schwarzschild, M. A. (2017). Targeting urate to reduce oxidative stress in Parkinson disease. Exp. Neurol. 298(Pt B), 210-224. doi: 10.1016/j.expneurol.2017.06.017

Dalrymple, A., Wild, E. J., Joubert, R., Sathasivam, K., Bjorkqvist, M., Petersen, A., et al. (2007). Proteomic profiling of plasma in Huntington's disease reveals neuroinflammatory activation and biomarker candidates. J. Proteome Res. 6, 2833-2840. doi: 10.1021/pr0700753

Davis, J. W., Grandinetti, A., Waslien, C. I., Ross, G. W., White, L. R., and Morens, D. M. (1996). Observations on serum uric acid levels and the risk of idiopathic Parkinson's disease. Am. J. Epidemiol. 144, 480-484. doi: 10.1093/ oxfordjournals.aje.a008954

Dorner, J. L., Miller, B. R., Barton, S. J., Brock, T. J., and Rebec, G. V. (2007). Sex differences in behavior and striatal ascorbate release in the 140 CAG knockin mouse model of Huntington's disease. Behav. Brain Res. 178, 90-97. doi: 10.1016/j.bbr.2006.12.004

Fang, P., Li, X., Luo, J. J., Wang, H., and Yang, X. F. (2013). A double-edged sword: uric acid and neurological disorders. Brain Disord. Ther. 2:109. 
Franco-Iborra, S., Vila, M., and Perier, C. (2018). Mitochondrial quality control in neurodegenerative diseases: focus on Parkinson's disease and Huntington's disease. Front. Neurosci. 12:342.

Gluhm, S., Goldstein, J., Brown, D., Van Liew, C., Gilbert, P. E., and CoreyBloom, J. (2013). Usefulness of the montreal cognitive assessment (MoCA) in Huntington's disease. Mov. Disord. 28, 1744-1747. doi: 10.1002/mds. 25578

Gonzalez, D., Marquina, R., Rondon, N., Rodriguez-Malaver, A. J., and Reyes, R. (2008). Effects of aerobic exercise on uric acid, total antioxidant activity, oxidative stress, and nitric oxide in human saliva. Res. Sports Med. 16, 128-137. doi: 10.1080/15438620802103700

Goula, A. V., Berquist, B. R., Wilson, D. M. III, Wheeler, V. C., Trottier, Y., and Merienne, K. (2009). Stoichiometry of base excision repair proteins correlates with increased somatic CAG instability in striatum over cerebellum in Huntington's disease transgenic mice. PLoS Genet. 5:e1000749. doi: 10.1371/ journal.pgen.1000749

Graham, S. F., Kumar, P., Bahado-Singh, R. O., Robinson, A., Mann, D., and Green, B. D. (2016). Novel metabolite biomarkers of Huntington's disease as detected by high-resolution mass spectrometry. J. Proteome Res. 15, 1592-1601. doi: 10.1021/acs.jproteome.6b00049

Granger, D. A., Fortunato, C. K., Beltzer, E. K., Virag, M., Bright, M. A., and Out, D. (2012). Focus on methodology: salivary bioscience and research on adolescence: an integrated perspective. J. Adolesc. 35, 1081-1095. doi: 10.1016/j.adolescence. 2012.01.005

Group, D. (1993). A novel gene containing a trinucleotide repeat that is expanded and unstable on Huntington's disease chromosomes. The Huntington.s Disease collaborative research group. Cell 72, 971-983. doi: 10.1016/0092-8674(93) 90585-e

Johri, A., and Beal, M. F. (2012). Antioxidants in Huntington's disease. Biochim. Biophys. Acta 1822, 664-674. doi: 10.1016/j.bbadis.2011.11.014

Jung, J. H., Song, G. G., Lee, Y. H., Kim, J. H., Hyun, M. H., and Choi, S. J. (2018). Serum uric acid levels and hormone therapy type: a retrospective cohort study of postmenopausal women. Menopause 25, 77-81. doi: 10.1097/GME. 0000000000000953

Kivity, S., Kopel, E., Maor, E., Abu-Bachar, F., Segev, S., Sidi, Y., et al. (2013). Association of serum uric acid and cardiovascular disease in healthy adults. Am. J. Cardiol. 111, 1146-1151. doi: 10.1016/j.amjcard.2012. 12.034

Li, X., Valencia, A., Sapp, E., Masso, N., Alexander, J., Reeves, P., et al. (2010). Aberrant Rab11-dependent trafficking of the neuronal glutamate transporter EAAC1 causes oxidative stress and cell death in Huntington's disease. J. Neurosci. 30, 4552-4561. doi: 10.1523/JNEUROSCI.5865-09. 2010

McFarland, N. R., Burdett, T., Desjardins, C. A., Frosch, M. P., and Schwarzschild, M. A. (2013). Postmortem brain levels of urate and precursors in Parkinson's disease and related disorders. Neurodegener Dis. 12, 189-198. doi: 10.1159/ 000346370

Paganoni, S., and Schwarzschild, M. A. (2017). Urate as a marker of risk and progression of Neurodegenerative Disease. Neurotherapeutics 14, 148-153. doi: 10.1007/s13311-016-0497-4

Politis, M., Lahiri, N., Niccolini, F., Su, P., Wu, K., Giannetti, P., et al. (2015). Increased central microglial activation associated with peripheral cytokine levels in premanifest Huntington's disease gene carriers. Neurobiol. Dis. 83, 115-121. doi: 10.1016/j.nbd.2015.08.011

Ribeiro, M., Rosenstock, T. R., Cunha-Oliveira, T., Ferreira, I. L., Oliveira, C. R., and Rego, A. C. (2012). Glutathione redox cycle dysregulation in Huntington's disease knock-in striatal cells. Free Radic. Biol. Med. 53, 1857-1867. doi: 10. 1016/j.freeradbiomed.2012.09.004

Riis, J. L., Bryce, C. I., Matin, M. J., Stebbins, J. L., Kornienko, O., Huisstede, L. V., et al. (2018). The validity, stability, and utility of measuring uric acid in saliva. Biomark Med 12, 583-596. doi: 10.2217/bmm-2017-0336
Schlesinger, I., and Schlesinger, N. (2008). Uric acid in Parkinson's disease. Mov. Disord. 23, 1653-1657. doi: 10.1002/mds.22139

Sun, Y., Zhang, H., Tian, W., Shi, L., Chen, L., Li, J., et al. (2019). Association between serum uric acid levels and coronary artery disease in different age and gender: a cross-sectional study. Aging Clin. Exp. Res. 31, 1783-1790. doi: 10.1007/s40520-019-01137-2

Towiwat, P., and Li, Z. G. (2015). The association of vitamin C, alcohol, coffee, tea, milk and yogurt with uric acid and gout. Int. J. Rheum. Dis. 18, 495-501. doi: 10.1111/1756-185X.12622

Trushina, E., and McMurray, C. T. (2007). Oxidative stress and mitochondrial dysfunction in neurodegenerative diseases. Neuroscience 145, 1233-1248. doi: 10.1016/j.neuroscience.2006.10.056

Ullah, M. F., Ahmad, A., Bhat, S. H., Abu-Duhier, F. M., Barreto, G. E., and Ashraf, G. M. (2019). Impact of sex differences and gender specificity on behavioral characteristics and pathophysiology of neurodegenerative disorders. Neurosci. Biobehav. Rev. 102, 95-105. doi: 10.1016/j.neubiorev.2019.04.003

van Dijk, J. G., van der Velde, E. A., Roos, R. A., and Bruyn, G. W. (1986). Juvenile Huntington disease. Hum. Genet. 73, 235-239.

Wen, M., Zhou, B., Chen, Y. H., Ma, Z. L., Gou, Y., Zhang, C. L., et al. (2017). Serum uric acid levels in patients with Parkinson's disease: a meta-analysis. PLoS One 12:e0173731. doi: 10.1371/journal.pone.0173731

Wexler, N. S., Lorimer, J., Porter, J., Gomez, F., Moskowitz, C., Shackell, E., et al. (2004). Venezuelan kindreds reveal that genetic and environmental factors modulate Huntington's disease age of onset. Proc. Natl. Acad. Sci. U.S.A. 101, 3498-3503.

Yang, Y., Zhou, W., Wang, Y., and Zhou, R. (2019). Gender-specific association between uric acid level and chronic kidney disease in the elderly health checkup population in China. Ren Fail 41, 197-203. doi: 10.1080/0886022x.2019. 1591994

Zagni, E., Simoni, L., and Colombo, D. (2016). Sex and gender differences in central nervous system-related disorders. Neurosci. J. 2016, 2827090. doi: 10. $1155 / 2016 / 2827090$

Zgaga, L., Theodoratou, E., Kyle, J., Farrington, S. M., Agakov, F., Tenesa, A., et al. (2012). The association of dietary intake of purine-rich vegetables, sugarsweetened beverages and dairy with plasma urate, in a cross-sectional study. PLoS One 7:e38123. doi: 10.1371/journal.pone.0038123

Zheng, J., Winderickx, J., Franssens, V., and Liu, B. (2018). A mitochondriaassociated oxidative stress perspective on Huntington's Disease. Front. Mol. Neurosci. 11:329. doi: 10.3389/fnmol.2018.00329

Zielonka, D., Marinus, J., Roos, R. A., De Michele, G., Di Donato, S., Putter, H., et al. (2013). The influence of gender on phenotype and disease progression in patients with Huntington's disease. Parkinsonism. Relat. Disord. 19, 192-197. doi: 10.1016/j.parkreldis.2012.09.012

Conflict of Interest: DG is the founder and Chief Scientific and Strategy Advisor at Salimetrics LLC and Salivabio LLC. The nature of these relationships is managed by the policies of the committees on conflict of interest at Johns Hopkins University School of Medicine and the University of California at Irvine. RF and SG were also employed by Salimetrics LLC.

The remaining authors declare that the research was conducted in the absence of any commercial or financial relationships that could be construed as a potential conflict of interest.

Copyright (C) 2020 Corey-Bloom, Haque, Aboufadel, Snell, Fischer, Granger, Granger and Thomas. This is an open-access article distributed under the terms of the Creative Commons Attribution License (CC BY). The use, distribution or reproduction in other forums is permitted, provided the original author(s) and the copyright owner(s) are credited and that the original publication in this journal is cited, in accordance with accepted academic practice. No use, distribution or reproduction is permitted which does not comply with these terms. 